\title{
Resonant Manipulation of $d$-wave Interaction of Cold Atoms with Two Lasers and a Magnetic Field
}

\author{
Bimalendu Deb \\ Department of Materials Science, Raman Center for Atomic, Molecular and Optical Sciences, \\ Indian Association for the Cultivation of Science, Jadavpur, Kolkata 700032, India.
}

\begin{abstract}
We present a theory for manipulation of $d$-wave interaction of cold atoms with two lasers strongly driving two photoassociative transitions. The theory predicts the occurrence of a coherence between two excited rovibrational bound states due to the photoassociative dipole-couplings of ground-state $d$-wave scattering state to the bound states. We show that this excited-state coherence significantly influences atom-atom interaction. In particular, this leads to the enhancement of $d$-wave elastic scattering and to the suppression of inelastic scattering. In the presence of an $s$-wave magnetic Feshbach resonance, the two lasers can couple the $s$-wave resonance with the $d$-wave scattering state leading to the further enhancement in $d$-wave scattering at relatively low energy. Our numerical calculations based on realistic parameters show that $d$-wave manipulation would be most effective in case of atoms having excited diatomic states with narrow natural linewidth. We estimate that at $100 \mu \mathrm{K}$ the inelastic scattering rate in $\mathrm{Yb}$ can be reduced to $20 \mathrm{~s}^{-1}$ while the elastic scattering rate can be two orders of magnitude larger.
\end{abstract}

PACS numbers: 67.10.Db,03.65.Nk,37.10.De,74.90.+n

\section{INTRODUCTION}

Cold atoms have now become a testing ground for interacting many-particle physics, in particular for models of condensed matter systems. They offer a unique opportunity to study many-body physics [1] with a controllable two-body interaction. By making use of the tunability of the $s$-wave scattering length with an external magnetic field, $s$-wave physics of interacting ultracold atoms has been extensively studied in recent times. To go beyond the $s$-wave physics, it is essential to devise methods for manipulation of higher partial-wave interactions of cold atoms. Higher partial-wave interactions are important for some exotic phases of matter such as the $p$-wave anisotropic superfluidity of liquid He-3 and the $d$-wave superconductivity of cuprate materials [2]. The ability to study phenomena related to $d$-wave Cooper pairing with fermionic atoms will help us to gain new insight into the open problem of high temperature superconductivity. To explore $d$-wave pairing of fermions with two spin components (like electrons) at various interaction regimes, it is of primary interest to devise an effective method of manipulating the $d$-wave interaction in two-component fermionic atoms.

There are mainly two methods of controlling atom-atom interaction at ultracold temperatures. The most widely used method involves the magnetic Feshbach resonance (MFR) [3, 4]. The other method using the optical Feshbach resonance (OFR) [5, 6] is a relatively recent one. Over more than a decade, the MFR has been used to study the $s$-wave physics of interacting atomic gases. In fact, the MFR has become a standard tool for studying Fermi superfluidity [7] and strongly interacting Fermi gases [8] with tunable $s$-wave scattering length. The OFR has been originally developed as a method for altering the $s$-wave scattering length as in the MFR. This method relies on off-resonant free-bound photoassociative transitions. The use of strong photoassociative coupling has been proposed as an optical method of manipulating higher partial-wave interactions [9]. Of late, $p$-wave OFR has been experimentally reported in fermionic ${ }^{171} \mathrm{Yb}$ atoms by Yamazaki et al. [10] based on a suggestion by
Goyal et al. [11] to use purely long-range states [12, 13] of photoassociated excited molecules. Earlier, magnetic field induced $p$-wave Feshbach resonances have been observed in spin-polarized ${ }^{40} \mathrm{~K} \mathrm{[14]}$ and ${ }^{6} \mathrm{Li}[15]$ Fermi gases. Not only $p$ wave OFR, but alo generating other higher partial-wave interactions in ultracold atoms under various physical conditions has attracted a great deal of research interest [16-20] in recent times. The generation of $d$-wave interaction in ultracold atoms by strong confinement has been discussed in Refs [18, 20]. However, an efficient manipulation of the $d$-wave atom-atom interaction for the purpose of studying $d$-wave many-body physics with cold atoms is yet to be demonstrated. Both $p$ - and $d$-wave scattering amplitudes of cold atoms are in general extremely small according to Wigner threshold laws. This poses a major challenge in manipulating $p$ - and $d$-wave interactions with an optical or any other field. The method we propose here is applicable for both $p$ - and $d$-wave manipulation, but in this work we specifically concentrate on $d$-wave only.

Here we present a theory for an all optical or magnetooptical manipulation of $d$-wave interaction in three dimensions. Consider two colliding ground-state cold atoms irradiated by two photoassociation (PA) lasers tuned near resonance with two excited ro-vibrational bound (molecular) states 1 and 2 characterized by two different rotational quantum numbers $J_{1}$ and $J_{2}$, respectively. However, these states may have the same or different vibrational quantum numbers. These rovibrational states must be accessible by PA transitions from the $d$-wave scattering state of the two ground-state atoms. The method we propose here relies on the quantum interference between the two PA transition pathways leading to the occurrence of a coherence between the two excited states. We show that this coherence causes suppression of inelastic scattering and enhancement of $d$-wave elastic scattering. Note that the inelastic scattering considered here is due to absorption from the PA lasers and loss due to spontaneous emission. Because of spontaneous emission from excited states, one-color OFR is bound to suffer from inelastic loss as discussed by Bohn and Julienne [21]. However, here we show that, by establishing a coherence between two excited ro-vibrational levels by two 

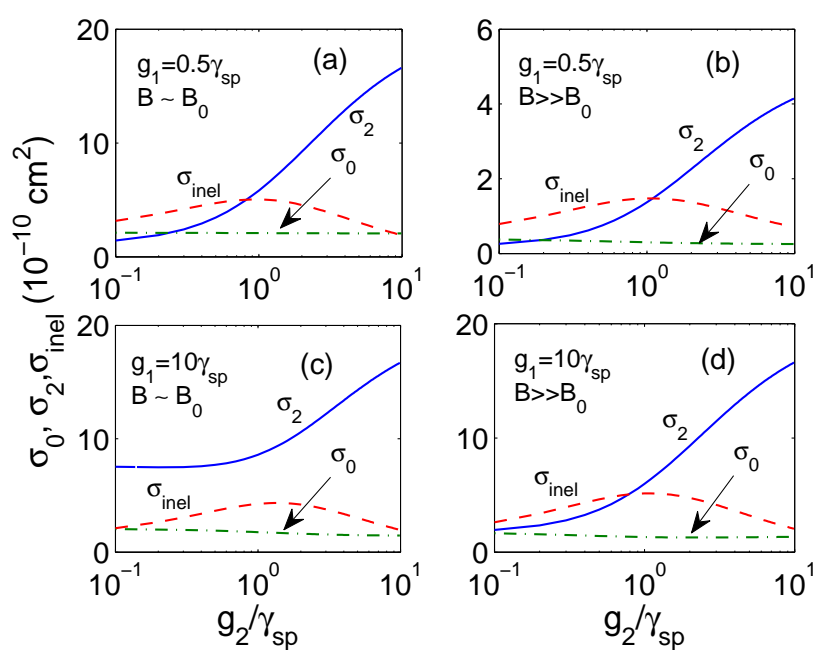

FIG. 1: Plots of $s$ - and $d$-wave elastic scattering cross sections $\sigma_{0}$ (dashed-dotted curves) and $\sigma_{2}$ (solid curves), respectively, and total inelastic scattering cross section $\sigma_{\text {inel }}$ (dashed curve ) for ${ }^{6} \mathrm{Li}$ atoms as a function of dimensionless scaled intensity $\left(g_{2} / \gamma_{s p}\right)$ of laser 2 for different values of the intensity of laser 1 and magnetic field: (a) $g_{1}=0.5 \gamma_{s p}$ and $\left(B-B_{0}\right) /|\Delta|=-0.24$, ( where $\Delta$ is the width of the MFR) (b) $g_{1}=0.5 \gamma_{s p}$ and $B \gg B_{0}$, (c) $g_{1}=10 \gamma_{s p}$ and $\left(B-B_{0}\right) / \Delta=-0.24$, and (d) $g_{1}=10 \gamma_{s p}$ and $B \gg B_{0} \cdot \gamma_{s p}$ is assumed to be $2 \mathrm{MHz}$ and energy $E=20 \mu \mathrm{K}$.

lasers, it is possible to suppress this inelastic loss significantly. Previous studies [22-24] on Fano-Feshbach resonances in PA [22] in the presence of a MFR [25-27] primarily focused on controlling $s$-wave scattering states, suppression of inelastic scattering [23] and control over power broadening [24]. The present work is basically concerned with optical manipulation of the anisotropic atom-atom interaction. In case of magnetic atoms, a magnetic field may be applied along the $z$ axis to induce an $s$-wave MFR. For two-component fermionic atoms or spin-polarized bosonic atoms, it is possible to optically couple the $s$-wave MFR to the $d$-wave scattering state by exploiting appropriate rotational selection rules for the two photoassociative transitions as analyzed below. The results of our numerical calculations based on realistic parameters reveal that the optical manipulation of the $d$-wave interaction is most effective in atoms having narrow natural linewidths such as $\mathrm{Yb}$.

\section{ANISOTROPIC DRESSED CONTINUUM}

Here we present a dressed state description of the groundstate continuum interacting with a magnetic field and a pair of laser fields.

\section{A. The model and its analytical solution}

For simplicity, let us consider only two ground-state scattering channels $\left|g_{1}\right\rangle$ and $\left|g_{2}\right\rangle$ with $\left|g_{1}\right\rangle$ being open (continuum) and $\left|g_{2}\right\rangle$ closed. To keep our discussions most general at the outset, we here do not specify the compositions of the channels, but we will give details of the channels in a specific numerical illustration later. The closed channel is assumed to support an $s$-wave bound state $|\chi\rangle$ whose binding energy lies close to the threshold of the $\left|g_{1}\right\rangle$ channel. These two ground-state channel thresholds and the closed-channel bound state may be tunable with an external magnetic field leading to an $s$-wave Feshbach resonance. The ground-state channels are asymptotically diagonal. However, at intermediate separations there is an interchannel coupling $V(r)$ which depends on the interatomic separation $r$.

The energy spacings between the excited bound states $\left|b_{1}\right\rangle$ and $\left|b_{2}\right\rangle$ characterized essentially by the two different rotational quantum numbers $J_{1}$ and $J_{2}$ are assumed to be large enough so that the two lasers $L_{1}$ and $L_{2}$ can drive only these two bound levels, respectively. If these two bound states belong to the same vibrational level $v$, then the rotational energy spacing between them must be large enough. Furthermore, these bound levels are assumed to be far below the respective dissociation threshold so that the transition probability at the single-atom level is negligible. The excited potentials asymptotically correlate to two separated atoms with one being in the electronic ground $S$ state and another in the excited $P$ state. If the two atoms are homonuclear, then the excited state potentials asymptotically go as $C_{3} / r^{3}$ where $C_{3}$ represents the resonant dipole-dipole interaction coefficient. In the case of heteronuclear atoms, the excited-state potential goes as $\sim-1 / r^{6}$ at large separations.

In the rotating wave approximation, the Hamiltonian of these three bound states interacting with the ground continuum can be expressed as $\hat{H}=\hat{H}_{0}+\hat{H}_{I}$ where

$$
\begin{aligned}
\hat{H}_{0} & =\sum_{n}\left(E_{n}-\hbar \omega_{L_{n}}\right)\left|b_{n}\right\rangle\left\langle b_{n}\right| \\
& +E_{\chi}|\chi\rangle\left\langle\chi|\otimes| g_{2}\right\rangle\left\langle g_{2}+\sum_{\ell m_{\ell}} \int E^{\prime} d E^{\prime}\right. \\
& \times\left|E^{\prime} \ell m_{\ell}\right\rangle_{\text {bare bare }}\left\langle E^{\prime} \ell m_{\ell}|\otimes| g_{1}\right\rangle\left\langle g_{1}\right|
\end{aligned}
$$

is the free part of the Hamiltonian with $E_{n}$ representing the binding energy of $n$-th excited molecular state $\left|b_{n}\right\rangle, E_{\chi}$ being the energy of the cosed-channel bound state $|\chi\rangle$ and $\mid$ $\left.E^{\prime}, \ell m_{\ell}\right\rangle_{\text {bare }}$ being the bare (unperturbed) partial-wave $\left(\ell m_{\ell}\right)$ scattering state with energy $E^{\prime}$. Note that all the energies are measured from the open-channel threshold unless stated otherwise. The interaction part of the Hamiltonian is

$$
\begin{aligned}
\hat{H}_{I} & =\sum_{n, M_{n}} \sum_{\ell m_{\ell}} \int d E^{\prime} \Lambda_{J_{n} M_{n}}^{\ell m_{\ell}}\left(E^{\prime}\right)\left|b_{n}\right\rangle\left\langle E^{\prime} \ell m_{\ell}\right|\left\langle g_{1}\right| \\
& +\int d E^{\prime} V_{\chi 0}\left(E^{\prime}\right)|\chi\rangle_{\text {bare }}\left\langle E^{\prime} 00|\otimes| g_{2}\right\rangle\left\langle g_{1}\right| \\
& +\sum_{n, M_{n}} \Omega_{n \chi}\left|b_{n}\right\rangle\langle\chi|\left\langle g_{2}\right|+\text { c.c. }
\end{aligned}
$$

Here $\Lambda_{\ell m_{\ell}}^{J_{n} M_{n}}(E)$ is the dipole matrix element of transition | $\left.b_{n}\right\rangle \rightarrow\left|E, \ell m_{\ell}\right\rangle_{\text {bare }}\left|g_{1}\right\rangle, V_{\chi 0}(E)$ is the coupling between the quasibound $(\chi)$ state and the $s$-wave $(\ell=0)$ scattering 
state of the bare continuum and $\Omega_{n \chi}$ is the Rabi frequency between the $n$th excited bound state and the $\chi$ state.

Let $|E, \hat{k}\rangle$ represent an eigen ket that satisfies the timeindependent Schrödinger equation $\hat{H}|E, \hat{k}\rangle=E|E, \hat{k}\rangle$, where $\hat{k}$ denotes a unit vector along the incident relative momentum of the two atoms. These eigen kets are energy normalized in a manner such that $\left\langle E^{\prime} \hat{k}^{\prime} \mid E, \hat{k}\right\rangle=\delta(E-$ $\left.E^{\prime}\right) \delta\left(\hat{k}-\hat{k}^{\prime}\right)$. To solve the Schrödinger equation, we expand $|E, \hat{k}\rangle$ in the following form

$$
\begin{array}{r}
|E, \hat{k}\rangle=\sum_{n=1,2} \sum_{M_{n}} A_{n E}\left|b_{n}\left(J_{n}, M_{n}\right)\right\rangle+B_{E}|\chi\rangle\left|g_{2}\right\rangle \\
+\sum_{\ell, m_{\ell}} \sum_{\ell^{\prime}, m_{\ell^{\prime}}} \int d E^{\prime} C_{E^{\prime}, \ell m_{\ell}}^{\ell^{\prime} m_{\ell^{\prime}}} Y_{\ell^{\prime} m_{\ell^{\prime}}}^{*}(\hat{k})\left|E^{\prime}, \ell m_{\ell}\right\rangle_{\text {bare }}\left|g_{1}\right\rangle
\end{array}
$$

where $B_{E}, A_{n E}$ and $C_{E^{\prime}, \ell m_{\ell}}^{\ell^{\prime} m_{\ell^{\prime}}}$ are the expansion coefficients to be derived. The coefficients $B_{E}$ and $A_{n E}$ can be expanded as $B_{E}=\sum_{\ell^{\prime} m_{\ell^{\prime}}} B_{E}^{\ell^{\prime} m_{\ell^{\prime}}} Y_{\ell^{\prime} m_{\ell^{\prime}}}^{*}(\hat{k})$ and $A_{n E}=$ $\sum_{\ell^{\prime} m_{\ell^{\prime}}} A_{n E}^{\ell^{\prime} m_{\ell^{\prime}}} Y_{\ell^{\prime} m_{\ell^{\prime}}}^{*}(\hat{k})$. Physically, $A_{n E}^{\ell^{\prime} m_{\ell^{\prime}}}$ implies the probability amplitude for the excitation of the bound state $\left|b_{n}\right\rangle$ when the incident partial-wave of relative motion of the two atoms is $\ell^{\prime}$ and its projection along the space-fixed $z$ axis is $m_{\ell^{\prime}}$. Similarly, $B_{E}^{\ell^{\prime} m_{\ell^{\prime}}}$ denotes the probability amplitude for the occupation of the quasibound state $|\chi\rangle$ for the $\left(\ell^{\prime} m_{\ell^{\prime}}\right)$ incident partial wave. The analytical expressions of the coefficients $A_{n E}^{\ell^{\prime} m_{\ell^{\prime}}}, B_{E}^{\ell^{\prime} m_{\ell^{\prime}}}$ and $C_{E^{\prime}, \ell m_{\ell}}^{\ell^{\prime} m_{\ell^{\prime}}}$ are derived in Appendix A. They are expressed in terms of the basic coupling parameters of the model, namely the laser couplings $\Lambda_{J_{n} M_{n}}^{\ell m_{\ell}}$ and magnetic coupling $V_{\chi 0}$. Explicitly,

$$
A_{n E}^{\ell^{\prime} m_{\ell^{\prime}}}\left(M_{n}\right)=\frac{\mathscr{A}_{n 0} \delta_{\ell^{\prime}, 0}+\mathscr{F}_{n \ell^{\prime}}\left(1-\delta_{\ell^{\prime} 0}\right)}{\mathscr{E}_{n}+i \hbar\left(\mathscr{G}_{n}+\gamma_{n}\right) / 2}
$$

The first term $\mathscr{A}_{n 0}$ in the numerator of Eq. (4) is defined by Eq. A25 in Appendix A. This term arises from incident partial wave $\ell^{\prime}=0$ ( $s$ wave) only. It consists of two terms. The first term $\beta_{n \epsilon} \Lambda_{J_{n} M_{n}}^{00}$ is the amplitude for transition form the $s$-wave scattering state to the $n$th bound state in the presence of a MFR and laser $L_{n}$. The effect of the MFR is given by the dimensionless parameter $\beta_{n \epsilon}=\frac{q_{n f}+\epsilon}{\epsilon+i}$. The parameter $q_{n f}$ is the Fano-Feshbach asymmetry parameter [24]. $\epsilon$ is a dimensionless energy parameter defined by Eq. (A19) and related to the MFR phase shift $\eta_{\text {res }}$ by the relation $\cot \eta_{\text {res }}=-\epsilon$. In the limit $\epsilon \rightarrow \pm \infty$ or equivalently $\eta_{\text {res }} \rightarrow 0$ or the coupling $V_{\chi 0} \rightarrow 0$ we have $\beta_{n} \rightarrow 1$. The second term $\frac{1}{\xi_{n^{\prime}}} \mathcal{K}_{n n^{\prime}} \beta_{n^{\prime} \epsilon} \Lambda_{J_{n^{\prime}} M_{n^{\prime}}}^{00}$ on the right-hand side (RHS) of Eq. A25) describes the combined effects of the other ( $n^{\prime}$ th) bound state, two lasers and the MFR on the transition amplitude from the $s$-wave scattering state to the $n$-th $\left(\neq n^{\prime}\right)$ bound state. The quantity $\mathcal{K}_{n n^{\prime}}=\mathcal{K}_{n n^{\prime}}^{\mathrm{mf}}+\mathcal{K}_{n n^{\prime}}^{\mathrm{LL}}$, where $\mathcal{K}_{n n^{\prime}}^{\mathrm{mf}}$ defined by Eq. A23 in Appendix A is an effective coupling between the two excited bound states induced by the two lasers in the presence of the MFR provided both the bound states are accessible by PA transitions from the $s$-wave scattering state. $\mathcal{K}_{n n^{\prime}}^{\mathrm{mf}}$ depends on the two Fano-Feshbach asymmetry parameters $q_{1 f}$ and $q_{2 f}$ and therefore on the optical dipole transitions from the closed channel $s$-wave quasibound state to the two excited states. $\mathcal{K}_{n n^{\prime}}^{\mathrm{LL}}$ given by Eq. A17 in Appendix A represents an effective coupling between the two excited states induced by the two lasers through continuum-bound PA transitions. Note that $\mathcal{K}_{n n^{\prime}}^{\mathrm{LL}}$ depends on the properties of the open-channel bare continuum and does not depend on closed-channel quasibound state or the bound-bound transition. All possible incident partial waves which are allowed by selection rules for both the PA transitions contribute to $\mathcal{K}_{n n^{\prime}}^{\mathrm{LL}}$. The second term $\mathscr{F}_{n \ell^{\prime}}$ in the numerator of Eq. (4) is given by Eq. (A26) in Appendix-A. This term describes transition amplitude from a nonzero incident partial wave $\left(\ell^{\prime}>0\right)$ to the $n$th bound state in the presence of both the lasers and the MFR. In the denominator of Eq. (4), $\mathscr{E}_{n}=E-\hbar \delta_{n}-E_{n}^{\text {shift }}-\mathscr{E}_{n} \mathrm{mf}-\mathscr{E}_{n n^{\prime}}$, where $E_{n}^{\text {shift }}$ is the light shift of $n$-th bound state due to $L_{n}$ laser only. $\mathscr{E}_{n} \mathrm{mf}$ given by Eq. A33 in Appendix A is a shift that strongly depends on the MFR, in the limit $\epsilon \rightarrow \pm \infty$ this shift vanishes. $\mathscr{E}_{n n^{\prime}}$ defined by Eq. (A34) in the Appendix-A depends on both lasers through the coupling $\mathcal{K}_{n n^{\prime}}$. The total linewidth for the transition to the $n$th bound state is $\mathscr{G}_{n}+\gamma_{n}$ where $\gamma_{n}$ is the spontaneous linewidth and $\mathscr{G}_{n}=\Gamma_{n 00}^{\operatorname{mf}}+\sum_{\ell>0, m_{\ell}} \Gamma_{n \ell m_{\ell}}+\Gamma_{n n^{\prime}}$ depends on the magnetic and laser fields. Here $\Gamma_{n 00}^{\mathrm{mf}}=\left|\beta_{n \epsilon}\right|^{2} \Gamma_{n 00}$ which, in the limit $\epsilon \rightarrow \pm \infty$ reduces to the $s$-wave partial stimulated linewidth $\Gamma_{n 00}$. Thus in the absence of the MFR, the first two terms in $\mathscr{G}_{n}$ can be added to yield $\Gamma_{n}=\sum_{\ell, m_{\ell}} \Gamma_{n \ell m_{\ell}}$ which is the stimulated linewidth of the $n$th bound state due to laser $L_{n}$. $\Gamma_{n n^{\prime}}$ is defined by Eq. A36 in Appendix A and explicitly depends on both lasers through $\mathcal{K}_{n n^{\prime}}$.

Finally, the remaining expansion coefficients of Eq. (3), the detailed derivation of which is given in the Appendix-A, can be expressed as

$$
\begin{aligned}
B_{E}^{\ell^{\prime} m_{\ell^{\prime}}} & =\frac{2}{(\epsilon+i) \Gamma_{f}}\left[V_{\chi 0}(E) \delta_{\ell^{\prime} 0}+\sum_{n, M_{n}}\left(q_{n f}-i\right)\right. \\
& \left.\times \pi \Lambda_{00}^{J_{n} M_{n}} V_{\chi 0}(E) A_{n E}^{\ell^{\prime} m_{\ell^{\prime}}}\right] \\
C_{E^{\prime} \ell m_{\ell}}^{\ell^{\prime} m_{\ell^{\prime}}}(E) & =\delta\left(E-E^{\prime}\right) \delta_{\ell \ell^{\prime}} \delta_{m_{\ell} m_{\ell^{\prime}}}+\frac{F_{\ell m_{\ell}}^{\ell^{\prime} m_{\ell^{\prime}}}\left(E, E^{\prime}\right)}{E-E^{\prime}}
\end{aligned}
$$

where

$$
\begin{aligned}
F_{\ell m_{\ell}}^{\ell^{\prime} m_{\ell^{\prime}}}\left(E, E^{\prime}\right) & =B_{E}^{\ell^{\prime} m_{\ell^{\prime}}} V_{\chi 0}\left(E^{\prime}\right) \delta_{\ell, 0} \\
& +\sum_{n, M_{n}} A_{n E}^{\ell^{\prime} m_{\ell^{\prime}}} \Lambda_{\ell m_{\ell}}^{J_{n} M_{n}}\left(E^{\prime}\right) .
\end{aligned}
$$

Equations (4), (5), (6) and (7) clearly show that all the expansion coefficients of Eq. (3) can be evaluated in terms of the known input parameters of our model. The effects of the two lasers and the magnetic field on atom-atom scattering are given by Eq. (7) as discussed in the next section. 


\section{B. Elastic and inelastic scattering}

Making use of the analytical solution as outlined above, we now derive scattering $T$ and $S$ matrices. The asymptotic form of the dressed continuum wave function $\langle\mathbf{r} \mid E, \hat{k}\rangle=$ $\Psi_{E}(\hat{k}, \mathbf{r})$, where $\mathbf{r}$ denotes relative coordinate, is given by $\Psi_{E}(\hat{k}, \mathbf{r} \rightarrow \infty) \sim r^{-1} \sum_{\ell m_{\ell}} \psi_{\ell m_{\ell}}(\hat{k}, r) Y_{\ell m_{\ell}}(\hat{r})$ where

$$
\begin{gathered}
\psi_{\ell m_{\ell}}(\hat{k}, r) \sim \sum_{\ell^{\prime} m_{\ell^{\prime}}}\left[\sin \left(k r-\frac{\ell^{\prime} \pi}{2}+\eta_{\ell^{\prime}}^{b g}\right) \delta_{\ell \ell^{\prime}} \delta_{m_{\ell} m_{\ell^{\prime}}}\right. \\
\left.-\pi F_{\ell m_{\ell}}^{\ell^{\prime} m_{\ell^{\prime}}}\left(E, E^{\prime}\right) Y_{\ell^{\prime} m_{\ell^{\prime}}}^{*}(\hat{k}) \exp \left[i\left(k r-\frac{\ell \pi}{2}+\eta_{\ell}^{b g}\right)\right]\right]
\end{gathered}
$$

with $\eta_{\ell^{\prime}}^{b g}$ being the background phase shift in the absence of interchannel and laser couplings. Now, equating the asymptotic form of $\Psi_{E}(\hat{k}, \mathbf{r})$ with the boundary condition $r^{-1} \sum_{\ell m_{\ell}}[\sin (k r-\ell \pi / 2+$ $\left.\left.\eta_{\ell}\right)-T_{\ell m_{\ell}}(\hat{k}) e^{i\left(k r-\ell \pi / 2+\eta_{\ell}\right)}\right] Y_{\ell m_{\ell}}(\hat{r})$ we deduce the anisotropic scattering $T$-matrix element $T_{\ell m_{\ell}}(\hat{k})=$ $\sum_{\ell^{\prime} m_{\ell^{\prime}}} T_{\ell m_{\ell}, \ell^{\prime} m_{\ell^{\prime}}}(E) Y_{\ell^{\prime} m_{\ell^{\prime}}}^{*}(\hat{k})$ where

$$
T_{\ell m_{\ell}, \ell^{\prime} m_{\ell^{\prime}}}(E)=T_{\ell^{\prime}}^{b g} \delta_{\ell \ell^{\prime}} \delta_{m_{\ell} m_{\ell^{\prime}}}+\pi F_{\ell m_{\ell}}^{\ell^{\prime} m_{\ell^{\prime}}}(E, E) e^{2 i \eta_{\ell}^{b g}}
$$

with $T_{\ell}^{b g}=-e^{i \eta_{\ell}^{b g}} \sin \left(\eta_{\ell}^{b g}\right)$ being the $T$-matrix element in the absence of all laser and magnetic couplings. The $S$-matrix element is obtained from $S_{\ell m_{\ell}}(\hat{k})=Y_{\ell m_{\ell}}(\hat{k})-2 i T_{\ell m_{\ell}}(\hat{k})$. The total scattering cross section can be obtained by means of the optical theorem as $\sigma_{t o t}=-\frac{4 \pi g_{s}}{k^{2}} \sum_{\ell m_{\ell}} \operatorname{Im}\left[T_{\ell m_{\ell}, \ell m_{\ell}}\right]$, where the symbol Im stands for the imaginary part and $g_{s}=$ 1 (2) for two distinguishable (indistinguishable) atoms. The total elastic scattering cross section $\sigma_{e l}=\sum_{\ell} \sigma_{\ell}$ where $\sigma_{\ell}=$ $\sum_{m_{\ell}} \sigma_{\ell m_{\ell}}$ and $\sigma_{\ell m_{\ell}}=\frac{4 \pi g_{s}}{k^{2}} \sum_{\ell^{\prime} m_{\ell^{\prime}}}\left|T_{\ell m_{\ell}, \ell^{\prime} m_{\ell^{\prime}}}\right|^{2}$. The total inelastic scattering cross section $\sigma_{\text {inel }}=\sigma_{t o t}-\sigma_{e l}$. Making use of Eq. (9), we have

$$
\sigma_{\text {inel }}=-\frac{4 \pi g_{s}}{k^{2}} \sum_{\ell m_{\ell}}\left[\Im\left\{\pi F_{\ell m_{\ell}}^{\ell m_{\ell}}\right\}+\sum_{\ell^{\prime}, m_{\ell^{\prime}}}\left|\pi F_{\ell m_{\ell}}^{\ell^{\prime} m_{\ell^{\prime}}}\right|^{2}\right]
$$

For both the spontaneous linewidths $\gamma_{1} \rightarrow 0$ and $\gamma_{2} \rightarrow 0$, we have $\sigma_{\text {inel }} \rightarrow 0$ and the $S$ matrix is unitary, that is $\sum_{\ell^{\prime} m_{\ell^{\prime}}} S_{\ell m_{\ell}, \ell^{\prime} m_{\ell^{\prime}}} S_{\ell^{\prime} m_{\ell^{\prime}}, \ell^{\prime \prime} m_{\ell^{\prime \prime}}}=$

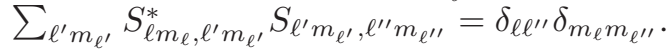

The first term on the RHS of Eq.99 is the isotropic part that does not depend on laser and magnetic fields. The second term proportional to $F_{\ell m_{\ell}}^{\ell^{\prime} m_{\ell^{\prime}}}(E, E)$ contains all the effects of laser and magnetic fields on scattering. The term $F_{\ell m_{\ell}}^{\ell^{\prime} m_{\ell^{\prime}}}(E, E)$ given by Eq. (7) is a sum of three terms corresponding to the three dressed bound-state amplitudes. For both lasers to influence $p$ - or $d$-wave scattering, it is essential that the rotational quantum numbers of the two excited bound states should be such that both of them are allowed by selection rules for PA transitions from the $p$ - or $d$-wave ground scattering state. Once bound state 1 or 2 is populated

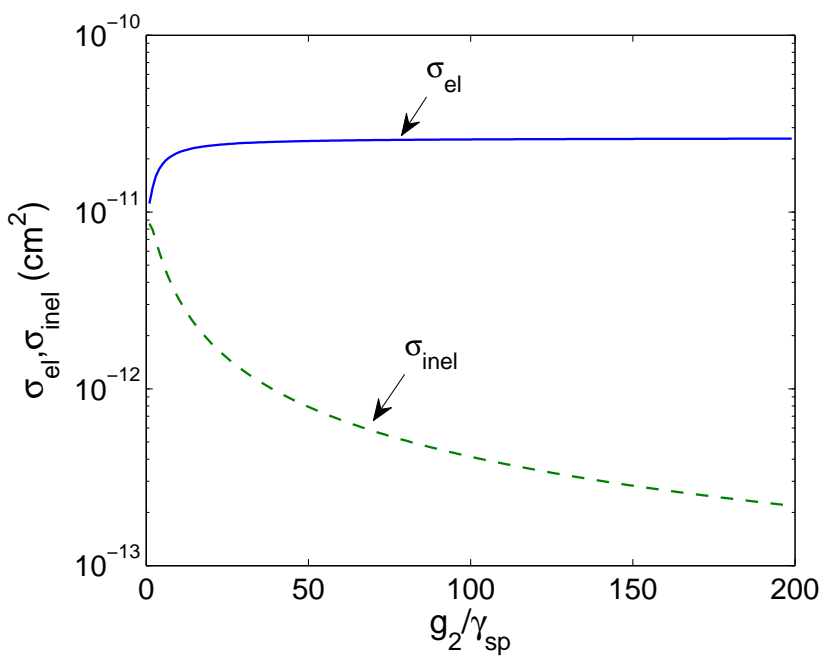

FIG. 2: This figure is for ${ }^{174} \mathrm{Yb}$ atoms. Plotted are the total elastic scattering cross section $\sigma_{e l}$ (solid) and total inelastic scattering cross section $\sigma_{\text {inel }}$ (dashed) as a function of $g_{2} / \gamma_{s p}$ for $g_{1}=200 \gamma_{s p}$ at $E=100 \mu \mathrm{K}$. The value of $\gamma_{s p}$ is $200 \mathrm{kHz}$.

or their coherent superposition is formed, a nonzero partialwave interaction in the ground continuum can be generated by two possible stimulated bound-free transition pathways. These pathways can interfere constructively under appropriate conditions resulting in enhanced higher partial-wave scattering amplitude. The sum of the two terms in $F_{\ell m_{\ell}}^{\ell^{\prime} m_{\ell^{\prime}}}(E, E)$ for $\ell \neq 0$ amounts to a coherent superposition of the amplitudes of those two transitions pathways. Furthermore, when a nonzero partial-wave ground scattering state is strongly coupled simultaneously to a pair of excited rovibrational states, a coherence between the two excited states due to the coupling $\mathscr{K}_{12}$ is generated. In the next section, we discuss how this coherence influences ground-state collisional properties.

\section{RESULTS AND DISCUSSIONS}

The analysis presented in the preceding section is quite general since it is applicable to any ultracold atomic gas. Equation (3) represents a nonperturbative eigenvalue solution of the model and is expressed as an anisotropic continuum dressed by two lasers and a magnetic filed. In the absence of the two lasers, the model reproduces the standard results of FanoFeshbach resonances as can be verified from Eqs. (9), (5), (6) and (7). In the absence of one laser and the magnetic field, the model reduces to that of $s$-wave [5] and (or) higher partial-wave OFR [9]. In what follows we apply this theoretical method to two specific atomic systems to show how the excited-state bound-bound coherence described by the term $\mathcal{K}_{n n^{\prime}}$ affects ground-state elastic and inelastic scattering properties.

For numerical illustrations, we consider two atomic systems: (A) fermionic ${ }^{6} \mathrm{Li}$ alkali atoms and (B) spin-singlet ground-state bosonic ${ }^{174} \mathrm{Yb}$ atoms. We assume that the two 
PA lasers $L_{1}$ and $L_{2}$ are co-propagating and linearly polarized in the $z$ axis for both systems. For this geometry, $\mathcal{K}_{n n^{\prime}} \neq 0$ only for $m_{\ell}=M_{1}=M_{2}$ for both systems.

\section{A. Fermionic ${ }^{6} \mathbf{L i}$ atoms}

To select appropriate excited states of ${ }^{6} \mathrm{Li}_{2}$ for our numerical illustrations, we consider low-lying rotational levels of a relatively deeply bound vibrational state of electronically excited $1^{3} \Sigma_{g}^{+}$potential which belongs to Hund's case b and asymptotically correlates to two separated $\left(S_{1 / 2}+P_{1 / 2}\right)$ atoms. We calculate several ro-vibrational states supported by this potential. The purpose is to choose two excited bound states which lie far below the dissociation threshold of the excited potential such that the possibility of laser transitions at the single-atom level can be safely ruled out. Furthermore, rotational energy spacings of such bound states should be large enough compared to other energy scales of our model. Hyperfine interaction in such excited bound states is assumed to be negligible compared to the resonant dipole-dipole interaction between the two atoms. The laser couplings $\Omega_{\chi n}$ and $\Lambda_{E, \ell m_{\ell}}^{J_{n} M_{n}}$ depend on the respective Franck-Condon (FC) overlap integral. To estimate FC integrals, we consider the $v=57$ vibrational state of the $1^{3} \Sigma_{g}^{+}$potential. This state has a vibrational energy $2575.3 \mathrm{GHz}$ below the dissociation threshold and an outer turning point around $30 a_{0}$. The rotational energy spacings $\Delta E_{v J}=E_{v, J+1}-E_{v, J}$ [where $E_{v, J}$ stands for the binding energy of the $(v, J)$ ro-vibrational state] are 3.4, 6.7 and $10.4 \mathrm{GHz}$ for $J=0, J=1$ and $J=2$, respectively. The rotational quantum number is given by $\vec{J}=\vec{N}=\vec{L}+\vec{\ell}$ where $L$ is the molecular electronic orbital angular momentum. The selection rule for laser transitions dictates that $\Delta N= \pm 1$.

Exchange symmetry of a pair of spin polarized ground state fermionic ${ }^{6} \mathrm{Li}$ atoms allows only odd partial waves in the ground continuum. In such a case, $p$-wave scattering state of the ground continuum can be optically coupled to both $J_{1}=0$ and $J_{2}=2$ rotational states by the two lasers. This can lead to the $p$-wave manipulation through optically generated coupling $\mathcal{K}_{n n^{\prime}}$ between the two excited rotational states.

Let us now consider two-component fermionic ${ }^{6} \mathrm{Li}$ alkali atoms initially prepared in hyperfine spin $f=1 / 2$ with two magnetic components $m_{f}=-m_{f}^{\prime}=1 / 2$. In the presence of a strong magnetic field $B$ (near $B \simeq B_{0}=834.1 \mathrm{G}$ for MFR), neither atomic hyperfine quantum number $f$ nor its projection $m_{f}$ along the quantization axis is a good quantum number, but the total magnetic quantum number $M_{F}=m_{f}+m_{f^{\prime}}$ of the two atoms with hyperfine numbers $f$ and $f^{\prime}$ remains a good quantum number at any magnetic field strength. In case of two-component ${ }^{6} \mathrm{Li}$ atoms with $M_{F}=0$, dipole selection rules allow $d$-wave to be coupled to both $J_{1}=1$ and $J_{2}=3$ rotational states by the two lasers while $s$-wave can be coupled to only $J_{1}=1$ rotational state. Henceforth, we focus only on $d$-wave manipulation.

To estimate ground-state input parameters, we calculate ground-state channel potentials in the presence of a magnetic field making use of the singlet and triplet ground-state potential data available in the literature [30, 31]. There are five asymptotic channels for $M_{F}=0$. We consider the lowest two asymptotic channels as a two-channel model for our numerical work. The method of calculation of ground-state channel potentials is described in some detail in Appendix B. Since the closed channel quasibound state $|\chi\rangle$ has $s$-wave rotational angular momentum, by selection rules we have $\Omega_{\chi n} \neq 0$ for $n=1$ and $\Omega_{\chi n}=0$ for $n=2$. However, both the $s$-wave bare continuum and the $|\chi\rangle$ state can be indirectly coupled to the $d$-wave via the $J=1$ bound state by two-photon process with both photons coming from laser $L_{1}$ in the strong-coupling PA regime. Furthermore, the rotational selection rule dictates $\Lambda_{00}^{J_{2} M_{2}}=0$ and hence $\mathcal{K}_{n n^{\prime}}=\mathcal{V}_{n n^{\prime}}-i \mathcal{G}_{n n^{\prime}} / 2$. Since $\mathcal{V}_{n n^{\prime}}$ is a small shift between the two excited states, for our numerical work we set $\mathcal{V}_{n n^{\prime}}=0$. Thus we have $\mathcal{K}_{n n^{\prime}}=-i \mathcal{G}_{n n^{\prime}} / 2$ where $\mathcal{G}_{n n^{\prime}}=2 \pi \Lambda_{J_{n} M_{n}}^{\ell=2 m_{\ell}} \Lambda_{\ell=2 m_{\ell}}^{J_{n^{\prime}} M_{n^{\prime}}}$ implying that this coupling term arises from the dipole interactions of the two excited bound states with the $d$-wave ground scattering state only.

Let us suppose that the frequencies of both lasers are tuned such that $E-\hbar \delta_{n}-E_{n}^{\text {shift }} \simeq 0$ for $n=1,2$. Then under the conditions mentioned above, the the numerator on the RHS of Eq. (4) becomes real provided the lasers do not introduce any phase as can be verified from Eqs. A25 and A26. It then follows from Eq. (10) that the terms that make positive contributions to $\sigma_{\text {inel }}$ are proportional to $\left(\mathscr{G}_{1}+\gamma_{1}\right) /\left|\mathcal{D}_{1}\right|^{2}$ or $\left(\mathscr{G}_{2}+\gamma_{2}\right) /\left|\mathcal{D}_{2}\right|^{2}$, where $\mathcal{D}_{n}=\mathscr{E}_{n}+i\left(\mathscr{G}_{n}+g_{n}\right) / 2$. Therefore, in order to suppress inelastic scattering, the terms $\mathscr{G}_{n}$ have to be reduced. Now, since $\mathscr{G}_{n}=\Gamma_{n 00}^{\mathrm{mf}}+\sum_{\ell>0 . m_{\ell}} \Gamma_{n \ell m_{\ell}}+\Gamma_{n n^{\prime}}$, and under the conditions $\Gamma_{n n^{\prime}}$ of Eq. A36 becomes equal to $-\left|\mathcal{G}_{n n^{\prime}}\right|^{2} /\left(\Gamma_{n^{\prime}}+\gamma_{n^{\prime}}\right)$ implying suppression of inelastic scattering due to optically induced coherence between the two excited bound states. Furthermore, since the $d$-wave $\left(\ell=2, m_{\ell}\right)$ elastic cross section is proportional to the term $\sum_{\ell^{\prime}, m_{\ell^{\prime}}}\left|F_{\ell=2 m_{\ell}}^{\ell^{\prime} m_{\ell^{\prime}}}\right|^{2}$ which is basically the summation of amplitudes for all the possible transitions from the two bound states to the $d$-wave ground continuum, the $d$-wave elastic scattering cross section would be enhanced when those transition amplitudes add up coherently or in other words, interfere constructively. The numerical results discussed below further corroborate these analytical findings.

For ${ }^{6} \mathrm{Li}$ atoms, the $s$-wave MFR is expected to influence the $d$-wave scattering process at relatively low energy. We find that for energies greater than $100 \mu \mathrm{K}$, the MFR has practically no influence. Therefore, for higher energies d-wave manipulation occurs mainly due to two lasers. This means that for higher energies our method of d-wave manipulation is essentially all optical. This optical method can be applicable for a wide range of energies in the sub millikelvin regime. We express the partial stimulated linewidth as $\Gamma_{n, \ell m_{\ell}}=g_{n} \mathscr{H}_{n, \ell m_{\ell}}$, where $g_{n}$ is the dipole coupling proportional to the intensity of the $n$th laser and $\mathscr{H}_{n, \ell m_{\ell}}$ is the angular part of the coupling. We assume that $\gamma_{1}=\gamma_{2}=\gamma_{s p}$ and scale all energy quantities by $\hbar \gamma_{s p}$. For all our numerical calculations, lasers $L_{1}$ and $L_{2}$ are assumed to be on resonance with PA transitions between the ground continuum and the excited bound states 1 and 2, respectively. For ${ }^{6} \mathrm{Li}$, an energy-dependent $s$-wave background phase shift is estimated from the experimental parameters of the MFR reported in Ref. [4] as discussed in Appendix B. For ${ }^{6} \mathrm{Li}$ atoms, the height of the $d$-wave centrifugal barrier is about 
$50 \mathrm{mK}$ and the peak of the barrier is located at a separation of around $50 a_{0}$. Therefore, the unperturbed $d$-wave scattering amplitude in the inner region of the barrier will be negligible unless the initial collision energy is relatively higher.

In Fig.1, we show $s$-wave and $d$-wave elastic and total inelastic scattering cross sections of ${ }^{6} \mathrm{Li}$ as a function of the intensity of the second laser for two different values of the intensity $\left(g_{1}\right)$ of the first laser at magnetic fields $B$ near resonant value $B_{0}$ [ Figs. 1(a) and 1(c)] and far away from the MFR [Figs. 1(b) and 1(d)]. Comparing Fig. 1(a) with Fig. 1(b) and Fig. 1(c) with Fig. 1(d), we infer that while the optical coupling of the MFR with the $d$-wave leads to significant enhancement of the $d$-wave scattering cross section in the strong-coupling regime, it has a marginal effect on inelastic scattering. It can also be noticed that the intensity of the second laser has almost no effect on the $s$-wave scattering amplitude for a fixed value of $B$. Comparing Fig.1(a) with Fig.1(c), we observe that with the increase of the intensity of the first laser, the $d$-wave scattering cross section increases when $B$ is tuned near the MFR. Since $J_{2}=3$ rotational state is not accessible from the $s$-wave scattering state, the freebound stimulated linewidth $\left(\Gamma_{n}\right)$ of the second $(n=2)$ bound state is almost entirely composed of $d$-wave partial stimulated linewidth. At $100 \mu \mathrm{K}$ temperature, in order to to make $\Gamma_{2} \simeq 1 \mathrm{MHz}$, the intensity of laser $L_{2}$ has to be about 500 $\mathrm{kW} / \mathrm{cm}^{2}$, while to make $\Gamma_{1} \simeq 1 \mathrm{MHz}$ in microkelvin temperature regime the intensity of laser $L_{1}$ is found to be of the order of $10 \mathrm{~kW} / \mathrm{cm}^{2}$. The unperturbed or background $d$-wave phase shift at $100 \mu \mathrm{K}$ temperature is found to be smaller than that of the $s$-wave background phase shift by four orders of magnitude. This means that the unperturbed $d$-wave scattering cross section in the microkelvin temperature regime is extremely small. Since the centrifugal barrier height is inversely proportional to the reduced mass of the two atoms, for heavier atoms the $d$-wave barrier height is lower. Therefore, in case of heavier atoms it might be possible to manipulate the $d$-wave interaction more efficiently with much lower laser intensities as shown in the next section.

\section{B. Bosonic ${ }^{174} \mathbf{Y b}$ atoms}

The method of manipulation of interatomic interaction in ${ }^{174} \mathrm{Yb}$ atomic gas is all optical because ${ }^{174} \mathrm{Yb}$ has no magnetic moment. For $d$-wave manipulation, as in the case of twocomponent ${ }^{6} \mathrm{Li}$ atoms, it is necessary to tune the two lasers $L_{1}$ and $L_{2}$ near the exited rotational levels $J_{1}=1$ and $J_{2}=3$, respectively. For the excited $\mathrm{Yb}_{2}$ molecule, $\vec{J}=\overrightarrow{J_{e}}+\vec{\ell}$ where $J_{e}$ is the total electronic angular momentum. The two excited states are assumed to belong to $0_{u}^{+}$(Hund's case c) molecular symmetry, that is, the projection of $J_{e}$ on the internuclear axis is zero. The $s$ - and $d$-wave background phase shifts and other input parameters for $\mathrm{Yb}$ are estimated from the data reported in Refs. [28, 29]. The ground-state potential of $\mathrm{Yb}$ is singlet only with no hyperfine structure and therefore the ground-state scattering of $\mathrm{Yb}$ is essentially single-channel.

We plot total elastic and inelastic scattering cross sections of ${ }^{174} \mathrm{Yb}$ as a function of $g_{2}$ in Fig.2. From this figure, we
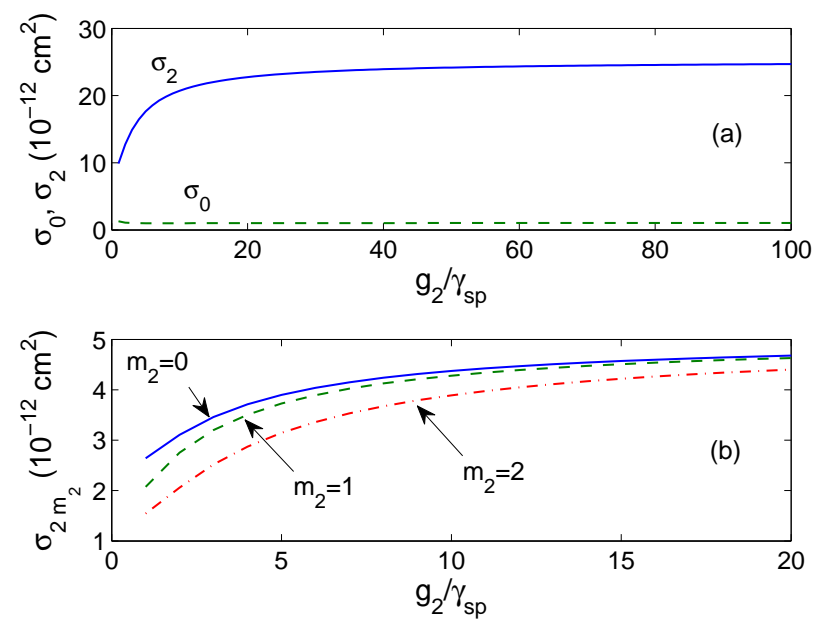

FIG. 3: These plots are for ${ }^{174} \mathrm{Yb}$ atoms. (a) $\sigma_{0}$ (dashed) and $\sigma_{2}$ (solid) are plotted as a function of $g_{2} / \gamma_{s p}$. (b) $\sigma_{2 m_{2}}$ Vs. $g_{2} / \gamma_{s p}$ for $m_{2}=0$ (solid), $m_{2}=1$ (dashed) and $m_{2}=2$ (dashed-dotted). The other parameters are the same as in Fig.2.

notice that for extreme strong-coupling regimes inelastic scattering can be suppressed by two orders of magnitude as compared to elastic scattering. $g_{2}=200 \gamma_{s p}$ corresponds to 40 MHz. From the theoretical results reported in Ref. [29], we estimate that at a laser intensity of $1 \mathrm{~W} / \mathrm{cm}^{2}$, the $d$-wave stimulated linewidth of ${ }^{174} \mathrm{Yb}$ at $100 \mu \mathrm{K}$ energy is about $0.1 \mathrm{MHz}$. Therefore, a 40-MHz stimulated linewidth will correspond to laser intensity of about $0.4 \mathrm{~kW} / \mathrm{cm}^{2}$. Since the spacing between the two excited bound states can be more than $50 \mathrm{MHz}$, the possibility of exciting multiple states by the laser 1 or laser 2 is unlikely.

We compare $s$ - and $d$-wave scattering cross sections in Fig. 3(a) which shows that the $d$-wave scattering cross section can exceed the $s$-wave one by an order of magnitude. Since $\mathcal{K}_{n n^{\prime}} \neq 0$ only for $m_{\ell}=M_{1}=M_{2}, \mathcal{K}_{n n^{\prime}}$ influences $d$-wave scattering for $-1 \leq m_{\ell=2} \leq 1$ only while $m_{\ell}= \pm 2$ remains unaffected by this term. Figure 3(b) demonstrates that $\sigma_{2 m_{2}}$ for $m_{2}=0$ is higher compared to those for $m_{2}=1$ and $m_{2}=2$ in the low $g_{2}$ regime but $\sigma_{2 m_{2}}$ for different $m_{2}$ values tend to merge and saturate in the high $g_{2}$ regime. This means that in the low $g_{2}$ regime the enhancement in $d$-wave scattering occurs predominantly with an interatomic axis oriented along $z$ axis or the axis of quantization.

From Fig. 2 we estimate the reduced inelastic rate of ${ }^{174} \mathrm{Yb}$ for a typical number density of $10^{13} / \mathrm{cm}^{3}$. Taking $\sigma_{\text {inel }} \sim$ $2 \times 10^{-13} \mathrm{~cm}^{2}$ and the velocity at $100 \mu \mathrm{K}$ temperature to be $\sim 10 \mathrm{~cm} / \mathrm{s}$, we find an inelastic rate $\sim 20 \mathrm{~s}^{-1}$. The elastic rate for $\sigma_{e l} \sim 10^{-11} \mathrm{~cm}^{2}$ would be two orders of magnitude larger than the inelastic rate. These numbers indicate that optical manipulation of $d$-wave interaction in $\mathrm{Yb}$ is possible. 


\section{CONCLUSIONS}

In conclusion, we have presented an analytical method for coherent manipulation of higher partial-wave atom-atom interaction by a pair of laser fields and an optional magnetic field in the strong photoassociative coupling regime. At low energy, this method is particularly applicable for manipulation of $p$ - and $d$-waves. Although, we have discussed $p$-wave manipulation, we have focused only on $d$-wave manipulation. The key effect predicted is the optically induced coherence between two rotational excitations. As a proof-of-principle, we have demonstrated the significance of this coherence in suppression of inelastic scattering and enhancement of $d$-wave elastic scattering. We have derived an analytical expression for the ground-state scattering $T$ matrix which clearly contains the effects of quantum interference. An analytical form of the two-body $T$ matrix is important as it may serve as a key ingredient for any theoretical treatment of interacting manyparticle systems. In this work, we have discussed application of our method to two-component fermionic ${ }^{6} \mathrm{Li}$ and bosonic ${ }^{174} \mathrm{Yb}$ atoms. However, our method is applicable to all the atomic species that are currently being used in cold atom research. It would be particularly interesting to apply our method to explore manipulation of $p$ - or $d$-wave interaction in heavier fermionic atoms such as ${ }^{171} \mathrm{Yb},{ }^{173} \mathrm{Yb}$ and ${ }^{87} \mathrm{Sr}$, all of which have very narrow linewidths. In order to make this method widely applicable, it is also important to find ways of reducing the natural linewidth of molecules by either tailoring vacuum fluctuations or by an interplay between light- and vacuum-induced coherences [32].

\section{Acknowledgments}

The author is thankful to C. Salomon, R. Hulet and Saikat Ghosh for discussions and comments.

\section{Appendix A: DERIVATION}

Here we present detailed derivation of the anisotropic dressed continuum of Eq. (3). From the time-independent
Schrödinger equation $\hat{H}|E, \hat{k}\rangle=E|E, \hat{k}\rangle$, making use of the expansions $B_{E}=\sum_{\ell^{\prime} m_{\ell^{\prime}}} B_{E}^{\ell^{\prime} m_{\ell^{\prime}}} Y_{\ell^{\prime} m_{\ell^{\prime}}}^{*}(\hat{k})$ and $A_{n E}=\sum_{\ell^{\prime} m_{\ell^{\prime}}} A_{n E}^{\ell^{\prime} m_{\ell^{\prime}}} Y_{\ell^{\prime} m_{\ell^{\prime}}}^{*}(\hat{k})$, we obtain the following set of coupled algebraic equations

$$
\begin{aligned}
\left(\hbar \delta_{n}-E\right) A_{n E}^{\ell^{\prime} m_{\ell^{\prime}}}+\Omega_{n \chi} B_{E}^{\ell^{\prime} m_{\ell^{\prime}}}= & -\sum_{\ell m_{\ell}} \int d E^{\prime} \Lambda_{J_{n} M_{n}}^{\ell m_{\ell}}\left(E^{\prime}\right) \\
& \times C_{E^{\prime} \ell m_{\ell}}^{\ell^{\prime} m_{\ell^{\prime}}}(E), \\
\left(E_{\chi}-E\right) B_{E}^{\ell^{\prime} m_{\ell^{\prime}}}+\sum_{n, M_{n}} \Omega_{\chi n} A_{n E}^{\ell^{\prime} m_{\ell^{\prime}}}= & -\int d E^{\prime} V_{\chi 0}\left(E^{\prime}\right) \\
& \times C_{E^{\prime} 00}^{\ell^{\prime} m_{\ell^{\prime}}}, \quad \text { (A2) }
\end{aligned}
$$

and

$$
\begin{aligned}
\left(E^{\prime}-E\right) C_{E^{\prime} \ell m_{\ell}}^{\ell^{\prime} m_{\ell^{\prime}}}(E) & +\sum_{n, M_{n}} \Lambda_{\ell m_{\ell}}^{J_{n} M_{n}}\left(E^{\prime}\right) A_{n E}^{\ell^{\prime} m_{\ell^{\prime}}} \\
& +V_{0 \chi}\left(E^{\prime}\right) B_{E}^{\ell^{\prime} m_{\ell^{\prime}}} \delta_{\ell 0}=0
\end{aligned}
$$

where $\delta_{n}=E_{n} / \hbar-\left(\omega_{L_{n}}-\omega_{A}\right)$ with $E_{n}$ being the binding energy of $n$-th excited bound state measured from the threshold of the excited state potential, $\omega_{L_{n}}$ is the laser frequency of $n$-th laser and $\omega_{A}$ the atomic transition frequency. Since the coefficient $C_{E^{\prime} \ell m_{\ell}}^{\ell^{\prime} m_{\ell^{\prime}}}$ is required to fulfill scattering boundary conditions at large separation of the two atoms, we can express Eq. $\mathrm{A3}$ in the form

$$
\begin{aligned}
C_{E^{\prime} \ell m_{\ell}}^{\ell^{\prime} m_{\ell^{\prime}}}(E) & =\delta\left(E-E^{\prime}\right) \delta_{\ell, \ell^{\prime}} \delta_{\ell^{\prime} m_{\ell^{\prime}}}+\frac{V_{0 \chi}\left(E^{\prime}\right)}{E-E^{\prime}} B_{E}^{\ell^{\prime} m_{\ell^{\prime}}} \delta_{\ell, 0} \\
& +\sum_{n, M_{n}} \frac{\Lambda_{\ell m_{\ell}}^{J_{n} M_{n}}\left(E^{\prime}\right)}{E-E^{\prime}} A_{n E}^{\ell^{\prime} m_{\ell^{\prime}}}
\end{aligned}
$$

The partial-wave symbols appearing in the superscript and subscript of $C_{E^{\prime} \ell m_{\ell}}^{\ell^{\prime} m_{\ell^{\prime}}}(E)$ refer to the incident and scattered partial waves, respectively. Substituting Eq. A4 in Eqs. A1 and $\mathrm{A} 2$, we obtain

$$
\begin{gathered}
\left(\delta_{n}-E\right) A_{n E}^{\ell^{\prime} m_{\ell^{\prime}}}+\Omega_{n \chi} B_{E}^{\ell^{\prime} m_{\ell^{\prime}}}=-\Lambda_{J_{n} M_{n}}^{\ell^{\prime} m_{\ell^{\prime}}}(E)-\left[\int d E^{\prime} \frac{V_{0 \chi}\left(E^{\prime}\right) \Lambda_{J_{n} M_{n}}^{00}\left(E^{\prime}\right)}{E-E^{\prime}}\right] B_{E}^{\ell^{\prime} m_{\ell^{\prime}}}-\sum_{\ell m_{\ell}}\left[\int d E^{\prime} \frac{\left|\Lambda_{\ell m_{\ell}}^{J_{n} M_{n}}\left(E^{\prime}\right)\right|^{2}}{E-E^{\prime}}\right] \\
\times A_{n E}^{\ell^{\prime} m_{\ell^{\prime}}}-\sum_{\ell m_{\ell}} \sum_{n_{n}}\left[\int d E^{\prime} \frac{\Lambda_{\ell m_{\ell}}^{J_{n} M_{n}}\left(E^{\prime}\right) \Lambda_{J_{n} M_{n^{\prime}}}^{\ell m_{\ell}}\left(E^{\prime}\right)}{E-E^{\prime}}\right] A_{n^{\prime} E}^{\ell^{\prime} m_{\ell^{\prime}}}, \\
\left(E_{\chi}-E\right) B_{E}^{\ell^{\prime} m_{\ell^{\prime}}}+\sum_{n, M_{n}} \Omega_{\chi n} A_{n E}^{\ell^{\prime} m_{\ell^{\prime}}}=-V_{\chi 0}(E) \delta_{\ell^{\prime} 0}-\int d E^{\prime} \frac{\left|V_{\chi 0}\left(E^{\prime}\right)\right|^{2}}{E-E^{\prime}} B_{E}^{\ell^{\prime} m_{\ell^{\prime}}}-\sum_{n, M_{n}} \int d E^{\prime} \frac{V_{\chi 0}\left(E^{\prime}\right) \Lambda_{00}^{J_{n} M_{n}}}{E-E^{\prime}} A_{n E}^{\ell^{\prime} m_{\ell^{\prime}}}(\mathrm{A}(6)
\end{gathered}
$$


$\hat{\epsilon} \cdot \hat{d}\left|J M^{\prime}\right\rangle \delta_{M, M^{\prime}}=\left|\left\langle\ell m_{\ell}|\hat{\epsilon} \cdot \hat{d}| J M\right\rangle\right|^{2}$, where $\hat{\epsilon}$ stands for laser polarization and $\hat{d}$ is a unit vector pointing toward the molecular dipole moment. Taking $E \rightarrow E+i \eta$ with $\eta=0^{+}$, we have

$$
\begin{aligned}
\int d E^{\prime} \frac{V_{0 \chi}\left(E^{\prime}\right) \Lambda_{J_{n} M_{n}}^{00}\left(E^{\prime}\right)}{E-E^{\prime}} & =V_{n \chi}^{(\ell=0)}(E)-i \pi V_{0 \chi}(E) \\
& \times \Lambda_{J_{n} M_{n}}^{00}(E)
\end{aligned}
$$

where

$$
V_{n \chi}^{(\ell=0)}(E)=\mathcal{P} \int d E^{\prime} \frac{V_{0 \chi}\left(E^{\prime}\right) \Lambda_{J_{n} M_{n}}^{00}\left(E^{\prime}\right)}{E-E^{\prime}}
$$

is an effective interaction between the closed-channel bound state $|\chi\rangle$ and the excited bound state $\left|b_{n}\right\rangle$ mediated through the $s$-wave part of the ground continuum. Similarly, we have

$$
\begin{gathered}
\int d E^{\prime} \frac{\left|V_{0 \chi}\left(E^{\prime}\right)\right|^{2}}{E-E^{\prime}}=E_{\chi}^{\text {shift }}-i \frac{\Gamma_{f}}{2}, \\
\int d E^{\prime} \frac{\left|\Lambda_{\ell m_{\ell}}^{J_{n} M_{n}}\left(E^{\prime}\right)\right|^{2}}{E-E^{\prime}}=E_{n \ell m_{\ell}}^{\text {shift }}-i \frac{\Gamma_{n \ell m_{\ell}}}{2},
\end{gathered}
$$

and

$$
\int d E^{\prime} \frac{\Lambda_{\ell m_{\ell}}^{J_{n} M_{n}}\left(E^{\prime}\right) \Lambda_{J_{n^{\prime}} M_{n^{\prime}}}^{\ell m_{\ell}}\left(E^{\prime}\right)}{E-E^{\prime}}=\mathcal{V}_{n n^{\prime}}^{\left(\ell m_{\ell}\right)}-i \frac{\mathcal{G}_{n n^{\prime}}^{\left(\ell m_{\ell}\right.}}{2}
$$

where

$$
E_{\chi}^{\text {shift }}=\mathcal{P} \int d E^{\prime} \frac{\left|V_{0 \chi}\left(E^{\prime}\right)\right|^{2}}{E-E^{\prime}}
$$

is the shift of the closed-channel bound state for its interaction with $s$-wave part of the open channel continuum, $\Gamma_{f}=$ $2 \pi\left|V_{0 \chi}(E)\right|^{2}$ is the magnetic Feshbach resonance line width, $E_{n \ell m_{\ell}}^{\text {shift }}=\mathcal{P} \int d E^{\prime} \frac{\left|\Lambda_{\ell m_{\ell}}^{J_{n} M_{n}}\left(E^{\prime}\right)\right|^{2}}{E-E^{\prime}}$ is the partial light shift and $\Gamma_{n \ell m_{\ell}}(E)=2 \pi\left|\Lambda_{\ell m_{\ell}}^{J_{n} M_{n}}(E)\right|^{2}$ is the partial stimulated line width of the $n$-th excited bound state. Here

$$
\begin{gathered}
\mathcal{V}_{n n^{\prime}}^{\left(\ell m_{\ell}\right)}=\mathcal{P} \int d E^{\prime} \frac{\Lambda_{\ell m_{\ell}}^{J_{n} M_{n}}\left(E^{\prime}\right) \Lambda_{J_{n^{\prime}} M_{n^{\prime}}}^{\ell m_{\ell}}\left(E^{\prime}\right)}{E-E^{\prime}}, \\
\mathcal{G}_{n n^{\prime}}^{\left(\ell m_{\ell}\right)}=2 \pi \Lambda_{\ell m_{\ell}}^{J_{n} M_{n}}(E) \Lambda_{J_{n^{\prime}} M_{n^{\prime}}}^{\ell m_{\ell}}(E) .
\end{gathered}
$$

Substituting Eqs. A7, A9, A10 and A11 into Eqs. (A5) and (A6), we obtain

$$
\begin{gathered}
\left(E-\delta_{n}-E_{n}^{\mathrm{shift}}+i \Gamma_{n} / 2\right) A_{n E}^{\ell^{\prime} m_{\ell^{\prime}}}-\left\{\Omega_{n \chi}+V_{n \chi}^{(\ell=0)}-i \pi \Lambda_{J_{n} M_{n}}^{00} V_{0 \chi}(E)\right\} B_{E}^{\ell^{\prime} m_{\ell^{\prime}}}=\Lambda_{J_{n} M_{n}}^{\ell^{\prime} m_{\ell^{\prime}}}(E)+\sum_{M_{n^{\prime}}} \mathcal{K}_{n n^{\prime}}^{\mathrm{LL}} A_{n^{\prime} E}^{\ell^{\prime} m_{\ell^{\prime}}} \\
\left(E-E_{\chi}-E_{\chi}^{\mathrm{shift}}+i \Gamma_{f} / 2\right) B_{E}^{\ell^{\prime} m_{\ell^{\prime}}}-\sum_{n, M_{n}}\left\{\Omega_{\chi n}+V_{n \chi}^{(\ell=0)}-i \pi \Lambda_{00}^{J_{n} M_{n}} V_{\chi 0}(E)\right\} A_{n E}^{\ell^{\prime} m_{\ell^{\prime}}}=V_{\chi 0}(E) \delta_{\ell^{\prime} 0}
\end{gathered}
$$

where $E_{n}^{\text {shift }}=\sum_{\ell m_{\ell}} E_{n \ell m_{\ell}}^{\text {shift }}$ is the total light shift due to laser $L_{n}, \Gamma_{n}=\sum_{\ell m_{\ell}} \Gamma_{n \ell m_{\ell}}$ is the corresponding total stimulated linewidth and

$$
\mathcal{K}_{n n^{\prime}}^{\mathrm{LL}}=\sum_{\ell m_{\ell}}\left(\mathcal{V}_{n n^{\prime}}^{\left(\ell m_{\ell}\right)}-i \frac{1}{2} \mathcal{G}_{n n^{\prime}}^{\left(\ell m_{\ell}\right.}\right)
$$

is an effective complex coupling between the two excited bound states $\left|b_{n}\right\rangle$ and $\left|b_{n^{\prime} \neq n}\right\rangle$ induced by the two lasers through optical dipole interactions. The superscript LL is used to indicate the use of two lasers. Equation A16 can be ex- pressed in a compact form

$$
\begin{aligned}
B_{E}^{\ell^{\prime} m_{\ell^{\prime}}} & =\frac{2}{(\epsilon+i) \Gamma_{f}}\left[V_{\chi 0}(E) \delta_{\ell^{\prime} 0}+\sum_{n, M_{n}}\left(q_{n f}-i\right)\right. \\
& \left.\times \pi \Lambda_{00}^{J_{n} M_{n}} V_{\chi 0}(E) A_{n E}^{\ell^{\prime} m_{\ell^{\prime}}}\right]
\end{aligned}
$$

where

$$
\epsilon=\frac{E-E_{\chi}-E_{\chi}^{\text {shift }}}{\Gamma_{f} / 2}
$$

is the dimensionless collision energy measured from the shifted binding energy $E_{\chi}+E_{\chi}^{\text {shift }}$ of the closed channel qua- 
sibound state $|\chi\rangle$ and

$$
q_{n f}=\frac{\Omega_{\chi n}+V_{n \chi}^{(\ell=0)}}{\pi \Lambda_{00}^{J_{n} M_{n}} V_{\chi 0}(E)}
$$

is the Fano-Feshbach asymmetry parameter that describes quantum interference between two transition pathways (i) $\left|b_{n}\right\rangle \stackrel{L_{n} \text { Laser }}{\longrightarrow}|\chi\rangle$ and (ii) $\left|b_{n}\right\rangle \stackrel{L_{n} \text { Laser }}{\longrightarrow} \mid E \ell=0 m_{\ell}=$ $0\rangle_{\text {bare }} \stackrel{\text { hf }}{\longrightarrow}|\chi\rangle$. Substituting Eq. (A18) into Eq. (A15), we have

$$
\begin{aligned}
& \left(E-\delta_{n}-E_{n}^{\mathrm{shift}}+i \frac{\Gamma_{n}}{2}-\frac{\left(q_{n f}-i\right)^{2}}{\epsilon+i} \frac{\Gamma_{n 00}}{2}\right) A_{n E}^{\ell^{\prime} m_{\ell^{\prime}}} \\
- & \sum_{M_{n^{\prime}}} \mathcal{K}_{n n^{\prime}}\left(M_{n}, M_{n^{\prime}}\right) A_{n^{\prime} E}^{\ell^{\prime} m_{\ell^{\prime}}}\left(M_{n^{\prime}}\right) \\
= & \frac{q_{n f}-i}{\epsilon+i} \Lambda_{J_{n} M_{n}}^{00}(E) \delta_{\ell^{\prime} 0}+\Lambda_{J_{n} M_{n}}^{\ell^{\prime} m_{\ell^{\prime}}}(E) .
\end{aligned}
$$

Here

$$
\mathcal{K}_{n n^{\prime}}\left(M_{n}, M_{n^{\prime}}\right)=\mathcal{K}_{n n^{\prime}}^{\mathrm{mf}}+\mathcal{K}_{n n^{\prime}}^{\mathrm{LL}}
$$

where

$$
\mathcal{K}_{n n^{\prime}}^{\mathrm{mf}}=\left(q_{n f}-i\right)\left(q_{n^{\prime} f}-i\right) \pi \Lambda_{J_{n} M_{n}}^{00} \Lambda_{00}^{J_{n^{\prime}} M_{n^{\prime}}} .
$$

Note that, when both the lasers are linearly polarized along the quantization axis, we have $\mathcal{K}_{n n^{\prime}}\left(M_{n}, M_{n^{\prime}}\right)=0$ unless $M_{n}=M_{n^{\prime}}$. Putting $n=1,2$ and $n^{\prime}=1,2$ with $n^{\prime} \neq n$ in Eq.(A17), one can write down two coupled equations which can be solved to obtain

$$
A_{n E}^{\ell^{\prime} m_{\ell^{\prime}}}\left(M_{n}\right)=\frac{\mathscr{A}_{n 0} \delta_{\ell^{\prime}, 0}+\mathscr{F}_{n \ell^{\prime}}\left(1-\delta_{\ell^{\prime} 0}\right)}{\mathcal{D}_{n}}
$$

where

$$
\begin{gathered}
\mathscr{A}_{n 0}=\beta_{n \epsilon} \Lambda_{J_{n} M_{n}}^{00}+\frac{1}{\xi_{n^{\prime}}} \mathcal{K}_{n n^{\prime}} \beta_{n^{\prime} \epsilon} \Lambda_{J_{n^{\prime}} M_{n^{\prime}}}^{00}, \\
\mathscr{F}_{n \ell^{\prime}}=\Lambda_{J_{n} M_{n}}^{\ell^{\prime} m_{\ell^{\prime}}}+\frac{1}{\xi_{n^{\prime}}} \mathcal{K}_{n n^{\prime}} \Lambda_{J_{n^{\prime}} M_{n^{\prime}}}^{\ell^{\prime} m_{\ell^{\prime}}}
\end{gathered}
$$

and

$$
\begin{aligned}
\mathcal{D}_{n}(E)=\xi_{n} & -\frac{\left(q_{n f}-i\right)^{2}}{\epsilon+i} \frac{\hbar \Gamma_{n 00}}{2} \\
& -\xi_{n^{\prime}}^{-1} \mathcal{K}_{n n^{\prime}} \mathcal{K}_{n^{\prime} n} .
\end{aligned}
$$

In Eq. A25, $\beta_{n \epsilon}$ is a dimensionless factor defined by

$$
\beta_{n \epsilon}=\frac{q_{n f}+\epsilon}{\epsilon+i}
$$

and in Eq. A27 $\xi_{n}(E)$ is given by

$$
\xi_{n}(E)=E-\hbar \delta_{n}-E_{n}^{\mathrm{shift}}+i \hbar\left[\gamma_{n}+\Gamma_{n}(E)\right] / 2
$$

In the above equations $n^{\prime} \neq n$ and $M_{n}=M_{n^{\prime}}$. We have here phenomenologically introduced the spontaneous linewidth $\gamma_{n}$ by making the replacement $\Gamma_{n} \rightarrow \Gamma_{n}+\gamma_{n}$. Writing Eq. A27) in the form

$$
\mathcal{D}_{n}(E)=\mathscr{E}_{n}+i \hbar\left(\mathscr{G}_{n}+\gamma_{n}\right) / 2,
$$

we have

$$
\begin{gathered}
\mathscr{E}_{n}=E-\hbar \delta_{n}-E_{n}^{\mathrm{shift}}-\mathscr{E}_{n}^{\mathrm{mf}}-\mathscr{E}_{n n^{\prime}} \\
\mathscr{G}_{n}=\Gamma_{n 00}^{\mathrm{mf}}+\sum_{\ell>0, m_{\ell}} \Gamma_{n \ell m_{\ell}}+\Gamma_{n n^{\prime}}
\end{gathered}
$$

where

$$
\begin{gathered}
\mathscr{E}_{n} \mathrm{mf}=\left[\frac{\left(q_{n f}^{2}-1\right) \epsilon-2 q_{n f}}{\epsilon^{2}+1}\right] \frac{\Gamma_{n 00}}{2} \\
\mathscr{E}_{n n^{\prime}}=\operatorname{Re}\left[\xi_{n^{\prime}}^{-1} \mathcal{K}_{n n^{\prime}} \mathcal{K}_{n^{\prime} n}\right] \\
\Gamma_{n 00}^{\mathrm{mf}}=\left|\beta_{n \epsilon}\right|^{2} \Gamma_{n 00} \\
\Gamma_{n n^{\prime}}=-\frac{2}{\hbar} \Im\left[\xi_{n^{\prime}}^{-1} \mathcal{K}_{n n^{\prime}} \mathcal{K}_{n^{\prime} n}\right]
\end{gathered}
$$

\section{Appendix B: GROUND STATE OF TWO-COMPONENT ${ }^{6} \mathrm{Li}$ ATOMS IN A MAGNETIC FIELD}

In the presence of a magnetic field, the angular basis states of the form $\left|f f^{\prime}, m_{f}, m_{f^{\prime}}, M_{F}\right\rangle$ (diabatic basis) of two separated atoms for different values of $f, f^{\prime}, m_{f}$ and $m_{f^{\prime}}$ get mixed up. The Hamiltonian of two ground state atoms at large separation in the presence of an external magnetic field is then diagonalizable in these diabatic basis and the eigenfunctions are regarded as the scattering channels. However, these are not diagonal at short separations where the Hamiltonian is diagonalizable in the molecular adiabatic basis. In the absence of hyperfine interactions, there are two adiabatic molecular potentials corresponding to the spin singlet and triplet, both of which asymptotically go as $\sim-1 / r^{6}$. The diabatic and adiabatic basis are related via transformation involving Wigner $9-j$ symbol. For $M_{F}=0$ there are five channels which become coupled at intermediate separations ( $\leq 30 a_{0}$, where $a_{0}$ is Bohr radius). The coupling is most significant at separations around $20 a_{0}$. We consider the two lowest asymptotic channels with $M_{F}=0$. The lower channel $\left|g_{1}\right\rangle$ is open and the upper channel $\left|g_{2}\right\rangle$ is closed. The asymptotic energy spacing between these two channels at $B_{0}$ is calculated to be $2.25 \mathrm{GHz}$. The open channel is mostly electronic spin triplet (about 90\% triplet) while the closed channel is largely spin singlet. Exchange symmetry of a pair of two-component fermionic atoms with $M_{F}=0$ allows only even partial waves of relative motion of the pair, while for a pair of single component fermionic atoms only odd partial waves are allowed. For ${ }^{6} \mathrm{Li}$ atomic gas initially prepared in a 50:50 mixture of two 
hyperfine components of $f=1 / 2$ and $M_{F}=0$, even partialwave atom-atom interactions are expected to be predominant.

At low energy, the Feshbach resonance width $\Gamma_{f}$ is given by $\Gamma_{f} / 2 \simeq k a_{b g} \Gamma_{0}$, where $k$ is the wave number related to the collision energy $E=\hbar^{2} k^{2} /(2 m)$ with $m$ being the reduced mass of the two atoms, $a_{b g}=-1405 a_{0}$ is the back ground scattering length, $\Gamma_{0}$ is a parameter related to the width $\Delta=-300 \mathrm{G}$ of the zero crossing by $\Delta=\frac{\Gamma_{0}}{\delta \mu}$, with $\delta \mu=2 \mu_{B}$ (where $\mu_{B}$ is the Bohr magneton) being the difference of magnetic moment of closedchannel quasibound state from that of the two free atoms in the open channel. The energy-dependence of the Feshbach resonance phase shift $\eta_{\text {res }}$ is given by $-\cot \eta_{\text {res }}=$ $\epsilon=\frac{E-E_{t h}}{\Gamma_{f} / 2}-\frac{E_{c}-E_{t h}}{\Gamma_{f} / 2}$, where $E_{t h}$ is the threshold of the open channel that depends on the applied magnetic field, and $E_{c}-E_{t h}=\delta \mu\left(B-B_{0}\right)$. The background $s$-wave phase shift (with effective range of background open channel potential assumed to be zero) $\eta_{0}^{b g}$ is given by $\cot \eta_{0}^{b g} \simeq-\frac{1}{k a_{b g}}$. Sine the coupling between open and closed channels occurs at an intermediate separation $(k r \ll 1)$, we can write $\Gamma_{f} / 2=$ $\pi\left|V_{\chi 0}\right|^{2}=\pi\left|\int d r \psi_{\chi}(r) V(r) \psi_{E, \ell=0}(r)\right|^{2} \simeq \frac{k a_{b g}}{1+\left(k a_{b g}\right)^{2}} \Gamma_{0}$, where $V(r)$ stands for coupling between the two channels and $\psi_{E, \ell=0}(r)=\langle r \mid E, \ell=0\rangle_{\text {bare }}$ is the energy normalized background (open channel) scattering state, which asymptotically behaves as $\psi_{E, 00}(r \rightarrow \infty) \sim \sin \left(k r+\eta_{0}^{b g}\right)$ and $\psi_{\chi}(r)=\langle r \mid \chi\rangle$
[1] I. Bloch, J. Dalibard, and W. Zwerger, Rev. Mod. Phys. 80, 885 (2008); S. Giorgini, L. P. Pitaevskii, and S. Stringari, ibid 80, 1215 (2008).

[2] J. G. Bednorz and K. A. Müller, Z. Phys. B: Condens. Matter 64, 189 (1986).

[3] E. Tiesinga, B. J. Verhaar, and H. T. C. Stoof, Phys. Rev. A 47, 4114 (1993).

[4] For a recent review on Feshbach resonances, see C. Chin, R. Grimm, P. Julienne, and E. Tiesinga, Rev. Mod. Phys. 82, 1225 (2010).

[5] P. O. Fedichev, Y. Kagan, G. V. Shlyapnikov and J. T. M. Walraven, Phys. Rev. Lett. 77, 2913 (1996).

[6] F. K. Fatemi, K. M. Jones and P. D. Lett, Phys. Rev. Lett. 85, 4462 (2000).

[7] M. W. Zwierlein et al., Nature (London) 435, 1047 (2005).

[8] O'Hara et al., Science 298, 2179 (2002); M. Greiner, C. A. Regal and D. S. Jin, Nature (London) 426, 537 (2003); S. Jochim et al. Science 302, 2101 (2003); M. W. Zwierlein et al., Phys. Rev. Lett. 91, 250401 (2003); M. Bartenstein et al., Phys. Rev. Lett. 92, 203201 (2004); J. Kinast et al., Phys. Rev. Lett. 92, 150402 (2004); T. Bourdel et al., Phys. Rev. Lett. 93, 050401 (2004).

[9] B. Deb and J. Hazra, Phys. Rev. Lett. 103, 023201 (2009).

[10] R. Yamazaki et al., e-print arXiv:1210.2567 (October 2012).

[11] K. Goyal, I. Reichenbach, and I. Deutsch, Phys. Rev A 82, 062704 (2010).

[12] K. Enomoto, M. Kitagawa, S. Tojo, and Y. Takahashi1, Phys. Rev. Lett. 100, 123001 (2008).

[13] I. Reichenbach, P. S. Julienne, and I. Deutsch, Phys. Rev. A 80. 020701 (R) (2009).

[14] C. A. Regal, C. Ticknor, J. L. Bohn, and D. S. Jin, Phys. Rev.
Lett. 90, 053201 (2003).

[15] J. Zhang et al., Phys. Rev. A 70, 030702 (2004).

[16] R. A. Williams et al. Science 335, 314 (2012).

[17] J. Levinsen, T. G. Tiecke, J. T. M. Walraven, and D. S. Petrov, Phys. Rev. Lett. 103, 153202 (2009).

[18] Y. Nishida and S. Tan. Phys. Rev. A 82, 062713 (2010).

[19] Q. Beaufils et al., Phys. Rev. A 79, 032706 (2009).

[20] P. Giannakeas, V. S. Melezhik, and P. Schmelcher, Phys. Rev. A 84, 023618 (2011).

[21] J. L. Bohn and P. S. Julienne, Phys. Rev. A 56, 1486 (1997).

[22] B. Deb and G. S. Agarwal, J. Phys. B: At. Mol. Opt. Phys. 42, 215203 (2009).

[23] B. Deb, J. Phys. B: At. Mol. Opt. Phys. 43, 085208 (2010).

[24] B. Deb and A. Rakshit, J. Phys. B: At. Mol. Opt. Phys. 42, 195202 (2009).

[25] Ph. Courteille, R. S. Freeland, D. J. Heinzen, F. A. van Abeelen, and B. J. Verhaar, Phys. Rev. Lett., 81, 69 (1998).

[26] V. Vuletic, C. Chin, A. J. Kerman, and S. Chu, Phys.Rev. Lett., 83, 943 (1999).

[27] M. Junker, D. Dries, C. Welford, J. Hitchcock, Y. P. Chen and R. G. Hulet, Phys. Rev. Lett. 101, 060406 (2008).

[28] K. Enomoto, M. Kitagawa, K. Kasa, S. Tojo, and Y. Takahashi1, Phys. Rev. Lett. 98, 203201 (2007).

[29] M. Borkowski, R. Ciurylo, P. S. Julienne, S. Tojo, K. Enomoto, and Y. Takahashi, Phys. Rev. Lett. 80, 012715 (2009).

[30] W. I. McAlexander et al., Phys.Rev. A 51, R871 (1995).

[31] W. T. Zemke and W. C. Stwalley, J. Chem. Phys. 97, 2053 (1993).

[32] S. Das, A. Rakshit and B. Deb, Phys. Rev. A 85, 011401(R) (2012). 